\title{
Evanescent Waves and Superluminal Behaviour of Matter
}

\author{
Luca Nanni* \\ Faculty of Natural Science, University of Ferrara, 44100 - Ferrara, Italy \\ *corresponding author e-mail: luca.nanni@student.unife.it
}

\begin{abstract}
Evanescent waves are non-propagating waves with imaginary wave vector. In this study we prove that they are solutions of the tachyon-like Klein-Gordon equation and that, in tunneling of ultrarelativistic spin-1/2 particles, they describe superluminal states arising from the interaction between the particle and the barrier. These states decay as the particle emerges from the opposite side of the potential barrier conserving the same initial energy but not necessarily the same mass. The obtained theory is applied to the neutrino with the purpose to explain the flavour oscillation during free flight and to determine the conditions that maximize the probability of its occurrence.
\end{abstract}

Keywords: Evanescent Waves; quantum tunneling; relativistic wave packet; neutrino; mass oscillation.

Pacs numbers: 03.65.Xp, 03.75.-b, 14.60.Pq.

\section{Introduction}

Evanescent waves are localized waves that have the property of resisting to diffraction in dispersive media, such as a potential barrier, even for long distances [1-8]. These non-propagating waves are characterized by an imaginary wave vector [1] and decay exponentially along the direction of propagation of the initial way impinging the barrier. In optics and in quantum mechanics the evanescent ways are associated to tunneling phenomena, where a wave packet bumps a potential barrier being dumped, to emerge from the opposite side with reduced amplitude but with the same energy of the incoming wave packet [9-12], as shown in Fig. 1: 


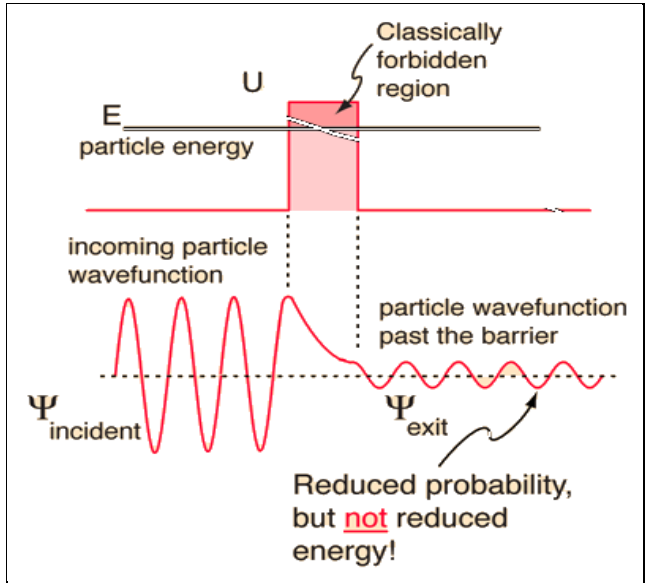

Figure 1: the incident way impinges the potential barrier and its amplitude decreases asymptotically. The outcome way emerges with reduced amplitude but same energy

Within the barrier the photon or the massive particle behaves like an evanescent wave that, if the barrier is enough large, is transmitted with superluminal group velocity [13-16]. This phenomenon is known as Hartman effect [17] and is based on the fact that the tunneling time, computed as Wigner phase time, does not depend on the barrier dimension [18-20]. In optics this effect has been experimentally verified [21] as well as in cosmology [22]. In particle physics, on the other hand, the Hartman effect has been extensively investigated but has not yet been supported by experimental evidences [23-25]. However, experimental confirmations could come from neutrino physics (superluminality and flavor oscillation) [26].

In this paper we will prove that the spinor evanescent wave is solutions of the tachyon-like Dirac equation (Lemke equation) [27] and that its components are solutions of the associated tachyon-like Klein-Gordon equation. This means that the evanescent waves in spinorial form are adequate to describe spin-1/2 particles in tachyonic regime. Then, we will investigate the behaviour of an ultrarelativistic spin-1/2 fermion in a potential barrier, highlighting that the tunneling can promote the particle into an excited state characterized by imaginary mass and tachyonic energy. Leaving the barrier, the particle returns in the ultrarelativistic regime, giving back the energy taken during the interaction with the barrier. During this process there is a non-zero probability that the fermion can change mass and momentum, always conserving the energy of the initial particle.

The obtained results will be applied to high-energy neutrino to explain its flavor oscillation during free flight. More precisely, we will determine the particle energy and the barrier dimension for which the probability that the neutrino changes its flavor is maximal. This theory is based on the fact that neutrino interacts with the surrounding matter so as to create that dispersive medium that acts as a potential barrier. If these interactions are enough intense, neutrino becomes superluminal making its flavor change possible. The problem is that neutrino interacts very weakly with matter and, therefore, the expected probability that it is in a superluminal excited state is very low but still different from zero. Therefore, this paper suggests that the study of the superluminal behaviour of neutrino may be done 
through the investigation of its flavor oscillation during free flight.

\section{Evanescent Ways and Tachyon-like Dirac Equation}

Within the potential barrier $U>E$, where $\mathrm{E}$ is the energy of the initial particle, the De Broglie wave vector is given by [8]:

$$
\chi=\frac{\sqrt{2 m(E-U)}}{\hbar}=\frac{i \sqrt{2 m(U-E)}}{\hbar} .
$$

Since such waves are associated with superluminal motions in tunneling phenomena, we have to prove that Eq. (1) is the wave vector of a tachyon, which is a particle with an imaginary mass. To do this we set the term $(U-E)$ equal to the tachyonic energy:

$$
(U-E)=\gamma_{t} m c^{2},
$$

where $\gamma_{t}$ is the tachyonic relativistic factor [28]:

$$
\gamma_{t}=\frac{1}{\sqrt{\left(\frac{u_{t}}{c}\right)^{2}-1}} \text { where } u_{t}>c \text {. }
$$

Replacing eq. (2) in (1) we get:

$$
\chi=\frac{i \sqrt{2 m\left(\gamma_{t} m c^{2}\right)}}{\hbar}=\frac{\sqrt{2 \gamma_{t}(i m)^{2} c^{2}}}{\hbar},
$$

which is just the De Broglie wave vector of a tachyonic plan wave related to a particle with imaginary mass $[21,29]$.

Let suppose that the free particle impinging the barrier is a monochromatic Dirac spinor; to further simplify the study we also suppose that the particle propagates along $x$-axis of the reference frame. In such a way there is not spin-flip and the spinor has only two components [30]. Each component of the Dirac spinor must satisfy separately the Klein-Gordon (KG) equation:

$$
|\psi\rangle=\left(\psi_{1}, \psi_{2}\right) \quad \Rightarrow \quad \partial_{x}^{2} \psi_{i}-\partial_{t}^{2} \psi_{i}=\left(\frac{m c}{\hbar}\right)^{2} \psi_{i}
$$

As known, for a free particle the solution of the $\mathrm{KG}$ equation are plan waves:

where $\omega$ is:

$$
\varphi(x, t)=\exp \{i(k x-\omega t)\}
$$

$$
\omega=c \sqrt{k^{2}+(m c / \hbar)^{2}} .
$$

If $|\omega|<\left(m c^{2} / \hbar\right)$ then the wave is evanescent in space [1]. We may use Eq. (7) to calculate $\omega$ in the case where the wave vector $k$ is that of a tachyonic particle:

$$
\omega_{t}=c \sqrt{\chi^{2}+(i m c / \hbar)^{2}}=c \sqrt{\left(1+2 \gamma_{t}\right)(i m c / \hbar)^{2}} .
$$

For a spin- $1 / 2$ free particle propagating along $x$-axis the Dirac spinor is [31]: 


$$
|\psi\rangle=\left(\begin{array}{c}
1 \\
\gamma \\
\gamma+1
\end{array}\right) \exp \{i(k x-\omega t)\} .
$$

where $\gamma$ is the Lorentz factor. Once the particle, in probabilistic terms, is within the potential barrier is described by the following evanescent spinor:

$$
\left|\varphi_{e v}\right\rangle=\left(\begin{array}{l}
1 \\
1
\end{array}\right) \exp \left\{\frac{m c}{\hbar}\left(1+2 \gamma_{t}\right)^{1 / 2}(c t-x)\right\},
$$

obtained by replacing $\chi$ and $\omega_{t}$ in Eq. (9). Since the evanescent wave is localized and does not propagate in time, the second component of the spinor becomes unitary and the temporal term $c t$ may be omitted. Moreover, considering that the particle exits from the barrier as a Dirac wave with dumped amplitude, it is reasonable to expect that the evanescent wave inside the barrier is compatible with a (virtual) tachyon with speed $u_{t}$ slightly higher than that of light.

We must now prove that the components of the spinor (10) are solutions of the tachyon-like $\mathrm{KG}$ equation, compatible with the momentum relation:

$$
E^{2}=p^{2} c^{2}-m^{2} c^{4}
$$

The equation is [27]:

$$
\partial_{x}^{2} \psi_{i}-\partial_{t}^{2} \psi_{i}=-\left(\frac{m c}{\hbar}\right)^{2} \psi_{i}
$$

Replacing in Eq. (11) the explicit form of each component of the evanescent wave (9), including eventually also the time part, we can easily verify the equality of right-hand-side and left-hand-side. We have thus shown that the evanescent wave describes the superluminal behaviour of matter in tunneling phenomena.

On the basis of the obtained results we can state that quantum tunneling brings the particle into a tachyonic state that from now on we define as localized excited state (virtual). This result is completely consistent with the Hartman effect, where the particle superluminality is estimated through the time needed to cross the potential barrier. This process occurs thanks to the interaction between the particle and the barrier: the more high the barrier is the greater the probability that the particle passes into a tachyonic state. This conclusion is consistent with the fact that the Hartman effect is particularly favoured by sufficiently large barriers [17, 23]; in this situation, in fact, the probability of interaction between the particle and the barrier increases, which is precisely the condition necessary to obtain a superluminal state.

\section{Tunneling of Ultrarelativistic Spin-1/2 Particle}

In the previous section for simplicity we have considered a monochromatic 
ultrarelativistic Dirac wave impinging a potential barrier. According to the uncertainty principle, however, a free particle propagating in space is represented by a wave packet that progressively spreads [24]. Therefore, for the continuation of this study we reconsider the tunneling phenomenon of an ultrarelativistic Dirac particle starting from a wave packet impinging a potential barrier with $U$ slightly higher than the initial particle energy. For this purpose we can choose an ultrarelativistic Gaussian wave packet, given a plane wave modulated by a Gaussian function [32]:

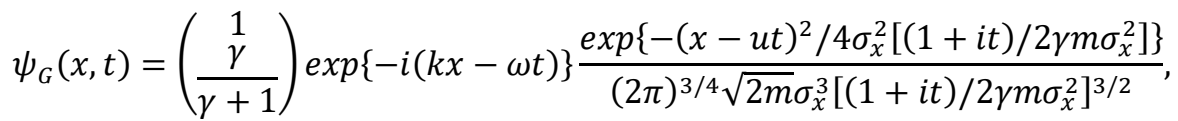

where $u$ is the initial particle velocity, $\gamma$ its Lorentz factor and $\sigma_{x}$ the spread indicator. As the Gaussian packet impinges the barrier, each plane wave that forms the envelope is reshaped in an evanescent wave [1]. Overall, within the barrier we have a monochromatic evanescent wave that can be represented as:

$$
\varphi_{G}(x, t)=\left(\begin{array}{l}
1 \\
1
\end{array}\right) e^{-\chi x} e^{-\omega t}
$$

As usual the temporal part of the evanescent wave may be omitted. If we denote with $x_{0}$ the point where the barrier begins, which length is $L$, in the space where $x<x_{0}$ the total spinor is given by the linear combination between the incident wave and the part of the wave that is reflected and does not penetrate the barrier [23]:

$$
\begin{aligned}
\psi_{x<x_{0}}(x, t)= & \left(\frac{1}{\gamma}\right) \Phi\left(u, \sigma_{x}\right)[\exp \{-i(k x-\omega t)\} \\
& \left.+c_{R} \exp \{i(k x-\omega t)\}\right],
\end{aligned}
$$

where $\Phi\left(u, \sigma_{x}\right)$ is the Gaussian function that appears in eq. (12). Within the barrier, i.e. $x_{0}<x<x_{0}+L$, the total evanescent spinor is given by the linear combination between the part of the impinging wave packet that has penetrated the barrier, and that is thus transformed in evanescent wave, and the part of the evanescent spinor that is reflected from the right side of the barrier:

$$
\varphi_{x_{0}<x<x_{0}+L}(x)=\left(\begin{array}{l}
1 \\
1
\end{array}\right)\left[\alpha e^{-\chi x}+\delta e^{\chi x}\right],
$$

where $\alpha$ and $\delta$ are numerical coefficients whose square represents a probability. Finally, beyond the barrier the spinor is represented by a wave packet with reduced amplitude:

$$
\psi_{x>x_{0}+L}(x, t)=c_{T}\left(\frac{1}{\gamma+1}\right) \Phi\left(u, \sigma_{x}\right) \exp \{-i(k x-\omega t)\},
$$

where $c_{T}$ is always a numerical coefficient whose square represents the probability that the ultrarelativistic particle is transmitted beyond the barrier. Eq. (16) tells us that the particle emerges from the barrier with the same energy that it had before the tunneling, but does not place constraints on the fact that momentum and rest 
mass may change. This peculiarity, even if speculative for what is the current knowledge on particle physics, is the point on which the rest of the theory will be developed.

Since each spinor component must be a continuous, smooth and derivable function, the following constraints must hold:

$$
\left\{\begin{array}{rl}
\psi_{G}\left(x=x_{0}, t\right) & =\varphi_{G}\left(x=x_{0}\right) \\
\psi^{\prime}{ }_{G}\left(x=x_{0}, t\right) & =\varphi^{\prime}{ }_{G}\left(x=x_{0}\right) \\
\psi_{G}\left(x=x_{0}+L, t\right) & =\varphi_{G}\left(x=x_{0}+L\right) \\
\psi_{G}^{\prime}\left(x=x_{0}+L, t\right) & =\varphi^{\prime}{ }_{G}\left(x=x_{0}+L\right)
\end{array} .\right.
$$

For the purpose of this work we are interested in calculating the probability that the particle penetrates the barrier and the probability that it is transmitted beyond the barrier; this means to calculate the coefficients $\alpha$ and $c_{T}$. To do this is necessary to solve the system of linear equations (17), introducing the explicit forms of spinors (14), (15), (16) and their derivatives. As far as the coefficient $\alpha$ is concerned, the calculation returns:

$$
\alpha=-2 \frac{\exp \left\{i m \sqrt{2 \gamma_{t}} c / \hbar\right\}\left[\gamma^{2}(c-u)^{2}+\gamma(c-u) \sqrt{2 \gamma_{t}} c\right]}{\left[\sqrt{2 \gamma_{t}} c-\gamma(c-u)\right]^{2}\left(e^{\chi L}-e^{-\chi L}\right)},
$$

If $L$ is large enough than function $e^{-\chi L}$ goes asymptotically to zero. Under this hypothesis the probability that the particle is within the barrier in a superluminal state is:

$$
|\alpha|^{2}=4 \frac{\left[\gamma^{2}(c-u)^{2}+\gamma(c-u) \sqrt{2 \gamma_{t}} c\right]^{2}}{\left[\sqrt{2 \gamma_{t}} c-\gamma(c-u)\right]^{4}} .
$$

From the conclusions obtained so far, we know the energy and therefore the velocity of the initial particle but not that concerning the superluminal state. To get the explicit form of probability (19) we must therefore express the tachyon Lorentz factor $\gamma_{t}$ as a function of the subluminal one. To do this, we equate Eqs. (1) and (4), representing different explicit forms of the tachyon wave vector; considering that $E=\gamma m c^{2}$ we get:

$$
\gamma_{t}=\frac{U}{m c^{2}}-\gamma
$$

Substituting Eq. (20) in Eq. (19) and passing, in order to simplify the notation, in natural units we obtain:

$$
|\alpha|^{2}=2 \frac{\gamma^{2}(1-u)^{2}}{(U-\gamma)}
$$

The probability with which the particle emerges from the barrier is [23]: 


$$
\left|c_{T}\right|^{2}=\frac{\gamma^{2}(1-u)^{2}(U-\gamma)}{\left[\gamma^{2}(1-u)^{2}+2(U-\gamma)\right] \sinh ^{2}(L \sqrt{2(U-\gamma)})} .
$$

Let us calculate now the ratio $\left|c_{T}\right|^{2} /|\alpha|^{2}$, that is the ratio between the probability that the particle emerges from the barrier and the probability that it is in a superluminal state:

$$
\frac{\left|c_{T}\right|^{2}}{|\alpha|^{2}}=\frac{(U-\gamma)^{2}}{2\left[\gamma^{2}(1-u)^{2}+2(U-\gamma)\right] \sinh ^{2}(L \sqrt{2(U-\gamma)})} .
$$

If $U$ is slightly higher than $E$ and the barrier is large enough, then:

$$
\lim _{\substack{U \rightarrow \gamma \\ L \rightarrow \infty}} \sinh ^{2}(L \sqrt{2(U-\gamma)}) \cong \sqrt{2} .
$$

Replacing this result in Eq. (23) and considering that $(U-\gamma) \cong 0$, we get (returning in conventional units):

$$
\frac{\left|c_{T}\right|^{2}}{|\alpha|^{2}}=\frac{(U-E)\left(U / m c^{2}-\gamma\right)}{2 \sqrt{2} \gamma^{2} m(c-u)^{2}} .
$$

We can see this relationship as the rate of production of an ordinary particle from a tachyon state in the time-lapse in which tunneling occurs. As above mentioned, the tunneling time does not depend on $L$, if the barrier is large enough. We find also this condition in the current theory of neutrino oscillation based on the hypothesis of flavors mixing [33]. In fact, in order to have the oscillation, the length $L$ must be greater than the wavelength of the flavor oscillation [34]. But if the barrier is large enough the tunneling is superluminal. Hence the fact that mass oscillation can occurs through a superluminal state. This explains why the study we are developing could help to investigate the neutrino flavor oscillation.

Let us go back to Eq. (20) and using the explicit forms of the subluminal and superluminal Lorentz factor we calculate the tachyon velocity associated with the particle state within the barrier:

$$
u_{t}^{2}=c^{2}\left[\frac{m c^{2}\left(c^{2}-u^{2}\right)}{U^{2}}\right] .
$$

In ultrarelativistic regime, where $u \cong c$, the tachyon velocity in the barrier is slightly higher than c. Under these conditions for a barrier with finite dimension and height $U<\infty$, the probability of penetration of the barrier is high enough but the tachyonic state that is formed is highly energetic. On the contrary, if $u \ll c$ then the tachyon velocity is higher than $c$ but the probability of penetration of the barrier is negligible. Finally, by setting an initial value of $u$ we can see that if $U$ is small enough, even if it is greater than $E$, then in term into brackets of Eq. (25) becomes relevant and the tachyonic velocity assumes values greater than $c$. 


\section{Neutrino Mass Oscillation by Superluminal Tunneling}

Let us apply now the obtained results to neutrino with the purpose of explaining the flavor oscillation during free flight. Assuming that $U$ is slightly higher than $E$, we can write:

$$
U=\eta \gamma m c^{2} \quad \text { with } \eta>1,
$$

that replaced in Eq. (24) gives:

$$
\frac{\left|c_{T}\right|^{2}}{|\alpha|^{2}}=\frac{c^{2}(\eta-1)^{2}}{2 \sqrt{2}(c-u)^{2}} .
$$

It has seen that, under the approximations made, this rate depends exclusively on the velocity of neutrino impinging the barrier and not on its mass. This means that each type of neutrino can give mass oscillation, even the massless one [35-36]. The greater the neutrino velocity and the barrier height are and the greater is the rate (26). It is evident that the barrier height depends on the ability of neutrino to interact with the matter it traverses. The greater the interactions are and the greater is the height of the equivalent barrier; these interactions [37], mediated by $W$ and $Z^{0}$ bosons, change the neutrino kinematics, from subluminal to tachyonic one. Because of these interactions, the neutrino cannot propagate as it does when it is in free space; everything happens as if neutrino crossed a potential barrier. The mediation of the weak interactions that in the quantum field theory is performed by $W$ and $Z^{0}$ bosons, in the relativistic quantum mechanics is performed by tunneling. But tunneling also describes superluminal behaviours that are not contemplated by the Standard Model, and this makes possible to study neutrino physics from an unconventional perspective, even if still speculative.

Let us now return to the probability that neutrino is transmitted beyond the potential barrier, given by Eq. (22). A fraction of this probability describes the neutrino retaining its flavor while, the complementary, is the probability that neutrino emerges from the barrier with different flavor:

$$
\left|c_{T}\right|^{2}=\rho\left|c_{T}\right|^{2}+(1-\rho)\left|c_{T}\right|^{2} \quad 0 \leq \rho \leq 1 .
$$

Since the relativistic energy of transmitted neutrino must be the same as the initial one, its change of mass involves a different momentum. But total moment must be conserved, and this means that the particle changes its momentum during the scattering that take place inside the barrier. This change, however, must be such that $p^{2} c^{2}>m^{2} c^{4}$, since the square of the tachyon energy must always be positive. Let us suppose $\rho\left|c_{T}\right|^{2}$ is the probability that neutrino changes flavour and, for simplicity, we consider the process $v_{\mu} \rightarrow v_{e}$ which takes place when muon neutrino interacts with matter. The initial state is described by the mixing of the two flavor 
states. If the matter density is constant, the probability that muon neutrino changes flavor is given by [38]:

$$
P=1-\sin ^{2}\left(2 \theta^{M}\right) \sin ^{2}\left(\frac{4 \pi L}{\lambda_{v}^{M}}\right)
$$

In Eq. (28) $\theta^{M}$ is the mixing angle in the matter while $\lambda_{v}^{M}$ the oscillation wave length in matter given by [38]:

$$
\lambda_{v}^{M}=\frac{4 \hbar \mathrm{cE}}{\Delta m^{2}} \frac{1}{\sqrt{\left(A / \Delta m^{2}-\cos 2 \theta\right)^{2}+\sin ^{2}(2 \theta)}} .
$$

The function $\sin ^{2}\left(2 \theta^{M}\right)$ is given by [38]:

$$
\sin ^{2}\left(2 \theta^{M}\right)=\frac{\sin (2 \theta)}{\sqrt{\left(A / \Delta m^{2}-\cos 2 \theta\right)^{2}+\sin ^{2}(2 \theta)}} .
$$

Finally, the numerical constant $A$ is:

$$
A=2 \sqrt{2}(\hbar \mathrm{c})^{3} G_{F} N_{e} E,
$$

where $G_{F}$ is the Fermi coupling constant, $N_{e}$ is the electron density in matter and $E$ is the neutrino initial energy.

Let us now rewrite explicitly the probability of transmission of neutrino beyond the barrier with flavour change, using all the approximations made in the previous section:

$$
\rho\left|c_{T}\right|^{2}=\rho \frac{U-E}{m c^{2}}=\rho(\eta-1) .
$$

By equating probability (31) with (28) and considering the resonance condition in which the amplitude of oscillation between the two flavor states is maximal (this means the constant $A$ must be equal to $\Delta m^{2} \cos 2 \theta$ ), we obtain:

$$
\rho(\eta-1)=1-\sin ^{2}\left(4 \pi L \sin (2 \theta) \frac{\Delta m^{2}}{4 \hbar \mathrm{cE}}\right) .
$$

Since $0 \leq \rho \leq 1$, it follows that the constraint on the numerical parameter $\eta$ to have resonance is:

$$
0 \leq \frac{1-\sin ^{2}\left(4 \pi L \sin (2 \theta) \Delta m^{2} / 4 \hbar \mathrm{cE}\right)}{\eta-1} \leq 1
$$

from which:

$$
\eta \geq 2-\sin ^{2}\left(4 \pi L \sin (2 \theta) \Delta m^{2} / 4 \hbar \mathrm{cE}\right) .
$$

We can then write the barrier energy as a function of the initial neutrino energy and the oscillation parameters:

$$
U \geq 2 E-E \sin ^{2}\left(4 \pi L \sin (2 \theta) \Delta m^{2} / 4 \hbar c E\right)
$$

Because the square sine function oscillates between zero and one, the probability 
that the superluminal neutrino changes flavor is constrained under the following conditions:

$$
\left\{\begin{array}{l}
\frac{L}{E}=\frac{K \hbar \mathrm{c}}{\left(2 \Delta m^{2} \sin 2 \theta\right)} \Rightarrow \rho=0 \\
\frac{L}{E}=\frac{2 K \hbar \mathrm{c}}{\left(2 \Delta m^{2} \sin 2 \theta\right)} \Rightarrow \rho \rightarrow 1
\end{array}\right. \text { where K is odd. }
$$

Eq. (35) provides the initial conditions for having a non-zero probability of neutrino mass oscillation during flight through matter. It is evident that the value of $L$ is essentially determined by the characteristics of the medium crossed by the neutrino, which must be such as to maximize its interaction with the surrounding electrons.

\section{Discussion}

To date, neutrino physics does not find the right place in the Standard Model; there are still too many open questions, well described in ref. [38], to which modern quantum field theory fails to give a satisfactory interpretation. Among these, the superluminal behaviour and the flavor oscillation are the most studied and discussed. Furthermore, superluminality and flavor oscillation behaviour arise from very similar experiments [39-42], which suggests that they are part of the same physical phenomenon [43-45]. This explains why other authors have tried to investigate these phenomena considering them intimately connected to each other [43-45].

The purpose of this study is to reconsider these ideas in the framework of relativistic quantum mechanics, taking advantage of the fact that neutrino interactions with matter can be assimilated to the effect of a potential barrier and that tunneling can lead to superluminal behaviours of particles. As proved, within the barrier the neutrino is superluminal and can decay in one of the possible flavor states. This hypothesis, even if based on speculative assumptions, does not violate the laws of quantum mechanics and has already been considered by other influential experts on this subject, even if concerning the mere study of superluminality [46]. Using a simplified model in which the initial state is a mix of two flavors, muon and electron neutrinos, we have obtained the ratio between the barrier width and the particle energy which assures the resonance condition such that the probability of flavor change is maximum. The problem is that even if these requirements are complied, the neutrino-matter interaction is too weak to be able to obtain an effective potential barrier for superluminal tunneling. This confirms that neutrino superluminality is a local environmental effect [47] and, at the same time, explains why flavor oscillation is a very rare phenomenon, especially for muon and tau neutrinos. In fact, from Eq. (32) we see that the probability of oscillation decreases as the term $\Delta m^{2}$ increases, explaining why the oscillations between tau and muon neutrino are not favourites. 
Finally, this theory suggests that the research of superluminal neutrino, whose few current experimental results have been denied, must be done precisely in the context of mass oscillation experiments. The measurement of the arrival time of a muon neutrino from an ultrarelativistic beam of electron neutrinos could effectively contain information on its possible superluminality.

\section{Conclusion}

In this study it has been shown that the bi-spinor wave packet, describing a free spin-1/2 particle in unidirectional motion, penetrating a potential barrier is transformed into an evanescent spinor. The components of this spinor are solutions of the tachyon-like Klein-Gordon equation, which proves that analytically the evanescent spinor describes the superluminal behaviour of the particle within the barrier. This behaviour is also confirmed by the fact that, if the barrier is enough large, the particle tunneling time does not depend on the barrier dimension (Hartman effect).

The obtained results have been applied to the study of the neutrino flavor oscillation. In particular, on the basis of the recent experimental results concerning ultrarelativistic neutrinos, up to energies of the order of the PeV [46], we propose a theory in which the flavor change takes place through the creation of a superluminal state obtained by the interaction of neutrino with the surrounding matter. This theory, still speculative, might be verified measuring the travel time between the source and the detector of the only high-energy neutrinos that arrive at the detector with changed flavor. This theory does not fall within the scope of quantum field theory, but it allows remedy part of the limits that actually prevent explaining the anomalous phenomena that characterize neutrino physics, such as flavor oscillation and superluminality.

\section{References}

1. E. Recami, M. Zamboni-Rached, Advances in Imaging and Electron Physics, Volume 156, 235, Elsevier (2009).

2. Z. Tong, O. Korotkova, Phys. Rev. A 85, 043802 (2012)

3. M. Xiao, Phys. Rev. E 60, 6226 (1999)

4. M. Milosevic, Appl. Spectrosc., 67(2), 126 (2013).

5. A.B. Katrich, Opt. Commun., 255, 169 (2005).

6. L.B. Felsen, J. Opt. Soc. Of Am., 66(8), 751 (1976).

7. T. Bertolotti, C. Sibilia, A.M. Guaman, Evanescent waves in optics: an introduction to plasmonics, Springer Verlag, Berlin (2017).

8. F. de Fornel, Evanescent waves from Newtonian optics to atomic optics, Springer, Berlin (2001).

9. A. Enders, G. Nimta, Phys. Rev. E 48, 632 (1993).

10. G. Agrawal, Nonlinear fiber optics, $4^{\text {th }}$ edition, Academic Press, New York (2006). 
11. A.M. Steinberg, P.G. Kwiat, R.Y Chiao, Phys. Rev. Lett., 71, 708 (1993).

12. C. Spielmann, R. Szipoc, A. Stingl, F. Krausz, Phys. Rev. Lett., 73, 2308 (1994).

13. S. Longhi, P. Laporta, M. Belmonte, E. Recami, Phys. Rev. E 65, 046610 (2002).

14. P. Ghose, M.K. Samal, Phys. Rev. E 64, 036620 (2001).

15. Z. Wang, C. Xiong, Phys. Rev. A 75, 042105 (2007).

16. D. Mugnai, A. Ranfagni, R. Ruggeri, Phys. Rev. Lett., 84, 4830 (2000).

17. T.E. Hartman, J. Appl. Phys., 33(12), 3427 (1962).

18. G. Muga, A. Raushhaupt, A. del Campo, Time in quantum mechanics, Volume 2, Springer, Berlin (2009).

19. P. Hrasko, Foundation of Physics, 33(7), 1009 (2003).

20. R. Landauer, Th. Martin, Rev. Mod. Phys., 66, 217 (1994).

21. E. Recami, J. Phys.: Conference Series, 196, 012020 (2009).

22. I.F. Mirabel, L.F. Rodriguez, Nature, 371, 4648 (1994).

23. L. Nanni, Open Phys., 15, 682 (2017).

24. M. Razavy, Quantum theory of tunneling, World Scientific, London (2003).

25. R. Kerner, R.B. Mann, Classical and Quantum Gravity, 25, 095014 (2008).

26. R. Ehrlich, Astropart. Phys., 41, 1 (2013).

27. H. Lemke, Il Nuovo Cimento, 35(2), 181 (1976).

28. E. Recami, Foudation of Physics, 17(3), 239 (1987).

29. R.K. Pradhan, L.P. Singh, https://arxiv.org/abs/0908.1365.

30. S.T. Park, Phys. Rev. A 86, 112 (2012).

31. Da-Wei Luo, P.V. Pyshkin, Ting Yu, Hai-Qing Lin, J.Q. You, Lian-Ao Wu, Phys. Rev. A 94, 032111 (2016).

32. D.V. Naumov, Phys. Part. Nuclei Lett,. 10, 642 (2013).

33. G. Cozzella, C. Giunti, Phys. Rev D 98, 096010 (2018).

34. K. Abe et al. (T2K Collaboration), Phys. Rev. D 88, 032002 (2013).

35. F. Benatti, R. Floreanini, Phys. Rev. D 64, 085015 (2001).

36. Hai-Long Zhao, https://arxiv.org/abs/1502.00691.

37. H. Spieceberger, M. Spira, P.M. Zerwas, https://arxiv.org/abs/hep-ph/0011255.

38. Z. Xing, S. Zhou, Neutrinos in Particle Physics, Astronomy and Cosmology, SpringerVerlag, Berlin (2011).

39. N. Agafonova et al., Phys. Rev. Lett. 121, 1399012018.

40. M. Antonello et al., Eur. Phys. J. C, 73, 2599 (2013).

41. A. Renshaw et al., Phys. Rev. Lett.112, 091805 (2014).

42. O. Botner, IceCube neutrino observatory: present and future, IceCube Particle Astrophysics Symp. (2015).

43. T.R. Morris, J. Phys. G: Nucl. And Part. Phys., 39045010 (2012).

44. K. Fujii, International Journal of Geometric Methods in Modern Physics, 10(2) 1250083 (2013).

45. B. Sazdović, M. Vasilić, J. High Energ. Phys, 2013: 47 (2013).

46. U.D. Jentschura, I. Nadori, R. Ehrlich, J. Phys. G: Nucl. Part. Phys,. 44, 105201 (2017).

47. G. Dvali, A. Vikman, J. High En. Phys., 2012: 134 (2012) 